\title{
Gemination or Fusion? A Diagnostic Dilemma
}

\section{Jain AA, Yeluri $\mathbf{R}^{*}$ and Munshi AK}

Department of Pedodontics and Preventive Dentistry, K.D Dental College and Hospital, Mathura, Uttar Pradesh, India

\begin{abstract}
In the primary dentition, little documentation of rare anomaly like fusion has been seen. This article presents the primary abnormalities of teeth i.e. Gemination and Fusion. It also highlights the presence of conjoined teeth in a 6 year old male child patient clinically presenting as gemination, but on radiographic evaluation turned out to be fusion. Clinically buccolingual groove with an increased mesio-distal coronal width was observed. Intraoral periapical radiograph revealed two fused crowns via dentine with single wide root canal and pulp chamber and the number of teeth in dental arch was less than the normal. So the clinico-radiologic correlation concluded it as fusion. Thus, it is very important for every dental professional to be familiar with dental developmental anomalies as their correct diagnosis would implicate a better prognosis.
\end{abstract}

Keywords: Double teeth; Developmental anomalies; Fusion; Gemination

\section{Gemination or Fusion? A diagnostic dilemma}

A six year old male child patient presented with increased mesiodistal coronal width of $9.5 \mathrm{~mm}$ along with the conjoined teeth in relation to the right mandibular central (81) and lateral (82) incisors (Figure 1). Clinical and radiographic examination revealed a distinct labio-lingual incisal groove; conclusive of either gemination of 81 with congenital missing 82 or fusion of 82 with 81 (Figure 2). No definitive treatment was carried out, as the tooth was clinically asymptomatic till date (patient was under regular follow up since last one year).

Development of the human dentition is a very complex process. The terms "double teeth", "double formation", "joined teeth", fused teeth, "dental twinning" are often used to describe gemination or fusion, both of which are primary developmental abnormalities of the teeth. Differential diagnosis is difficult; therefore several authors use the term 'double teeth'. The etiology of fusion is still unknown; however, the crowding of the tooth germs during their development can be an important factor. Local developmental aberrations of ectoderm and mesoderm during morpho-differentiation of tooth bud can be considered as etiological factors [1]. Genetic predisposition and racial differences have also been reported as contributing factors [2]. They do not show any sex predilection but are more frequent among Japanese population and American Indians [3]. Knezevic et al. [4] examined 3517 plaster models in order to find the prevalence of double teeth (fusion and gemination) among the individuals tested based on gender, distribution in the maxilla or mandible, and whether the anomaly occurred bilaterally or unilaterally. The results of their investigation on

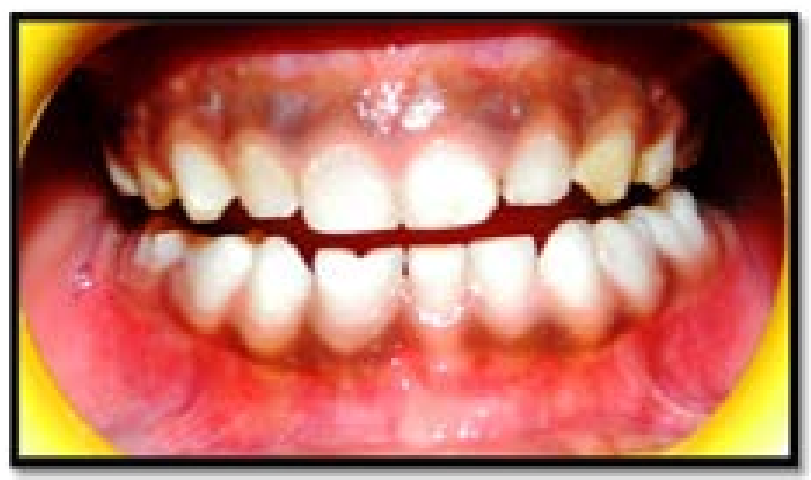

Figure 1: Mandibular right central (81) and (82) incisor presented with conjoined teeth a prevalence of double teeth appeared as $0.2 \% ; 57.2 \%$ of them fused and $42.9 \%$ geminated. Yuen et al. [5] have revealed that the fusion of primary teeth is associated mostly with hypodontia in succedaneous dentition. A suggested scheme of classification is as follows: Gemination (cleavage of a single tooth germ) - partial cleavage (true-gemination); complete cleavage (twinning). Fusion (two separate tooth germs fused during formative stage) - union by enamel and dentin (true fusion); union by dentin and/or cementum (late fusion); a late fusion by cementum is called a concrescence [6].

Despite the considerable number of cases reported in the literature,

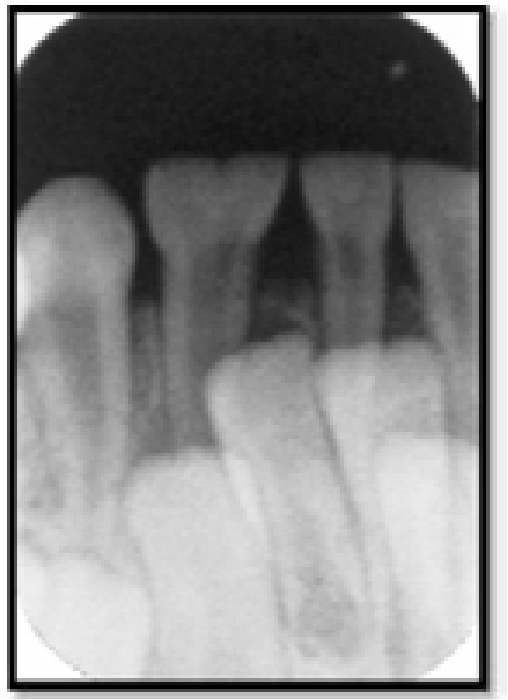

Figure 2: IOPA showing a distinct labio-lingual incisal groove with 81 .

${ }^{*}$ Corresponding author: Dr. Ramakrishna Yeluri. M.D.S, F.P.F.A, Professor Department of Pedodontics and Preventive Dentistry, K.D. Dental College and Hospital, Mathura-Delhi N.H \#2, Mathura-281001, Uttar Pradesh, India, Tel: +919997951558; Fax: 0565-2530764; E-mail: drramakrishnay@gmail.com, kittypedo@yahoo.com

Received December 17, 2013; Accepted Janaury 13, 2014; Published January 15,2014

Citation: Jain AA, Yeluri R, Munshi AK (2014) Gemination or Fusion? A Diagnostic Dilemma. Dentistry 4: 196. doi: 10.4172/2161-1122.1000196

Copyright: @ 2014 Jain AA, et al. This is an open-access article distributed unde the terms of the Creative Commons Attribution License, which permits unrestricted use, distribution, and reproduction in any medium, provided the original author and source are credited. 
the differential diagnosis between these abnormalities is difficult. Case history and clinical and radiographic examinations can provide the information required for the diagnosis of such abnormalities. Mader [7] proposed the "TWO TOOTH RULE" which may be a practical way of differentiating between fusion and gemination. If fused tooth are counted as one and the number of teeth in the dental arch is less, then the term fusion is considered. In our case we observed that 82 were fused with 81 and the number of teeth in the dental arch was found to be less. Also in clinico-radiologic correlation a single wide pulp chamber and root canal was found, thus it was conclusive of fusion. However, when the abnormal tooth is counted as one and the number of teeth in the dental arch is normal then it is termed as gemination or is a case of fusion between a normal and supernumerary teeth. Since the course of odontogenesis cannot be witnessed fusion and germination seem to be equivalent.

The complications that follow a fusion are many. The teeth affected are morphologically abnormal and so unaesthetic [2]. The buccal and lingual grooves may be deep and extend subgingival favoring plaque accumulation leading to dental caries and periodontal diseases [2]. The complex tooth morphology and pulpal anatomy, tooth position, and difficulty in rubber dam placement may negate endodontic treatment and necessitate surgical removal of the affected tooth. Fusion of primary teeth may lead to hypodontia, malformation, and impaction, delayed, or altered path of eruption of permanent successors [3]. Asymptomatic anterior fusion should be left alone unless problems arise with esthetics, spacing, and dental caries. Simple composite restorations can be used to camouflage and prevent caries developing in the fissure. Early diagnosis of the anomaly has a considerable importance. It should be followed by careful clinical and radiographic observations. Thus, it is very important for every dental professional to be familiar with dental developmental anomalies as these abnormal morphology demands prophylactic and early interceptive treatment in order to avoid the complicated pulpal and periodontal treatment related to these teeth.

\section{References}

1. Tsesis I, Steinbock N, Rosenberg E, Kaufman AY (2003) Endodontic treatment of developmental anomalies in posterior teeth: treatment of geminated/fused teeth-report of two cases. Int Endod J 36: 372-379.

2. Nunes E, de Moraes IG, de Novaes PM, de souse SM (2002) Bilateral fusion of mandibular second molars with supernumerary teeth: Case report. Braz Dent J 13: 137-141

3. Wu CW, Lin YT, Lin YT (2010) Double primary teeth in children under 17 years old and their correlation with permanent successors. Chang Gung Med J 33: 188-193.

4. Knezevic A, Travan S, Tarle Z, Sutalo J, Jankovic B, et al. (2002) Double tooth Coll Antropol 26: 667-672.

5. Yuen SW, Chan JC, Wei SH (1987) Double primary teeth and their relationship with the permanent successors: a radiographic study of 376 cases. Pediatr Dent 9: 42-48.

6. Tannenbaum KA, Alling EE (1963) Anomalous tooth development: case reports of germination and twinning. Oral Surg Oral Med Oral Pathol 16: 883-887.

7. Mader CL (1979) Fusion of teeth. J Am Dent Assoc 98: 62-64. 